Arthroskopie $2011 \cdot 24: 93-94$ DOI 10.1007/s00142-011-0636-1

Online publiziert: 15. Mai 2011

(c) Springer-Verlag 2011

\author{
K.P. Benedetto ${ }^{1}$ M. Cejna ${ }^{2}$ \\ ${ }^{1}$ Abteilung für Unfallchirurgie und Sporttraumatologie, \\ Universitäres Lehrkrankenhaus Feldkirch \\ ${ }^{2}$ Institut für diagnostische und interventionelle Radiologie, \\ Universitäres Lehrkrankenhaus Feldkirch
}

\title{
Bildgebung vor und nach arthroskopischen Eingriffen
}

Sehr geehrte Leserinnen und Leser,

die Arthroskopie stellt nach wie vor den Goldstandard zur Beurteilung der rein intraartikulären Pathologien der verschiedenen Gelenke dar. Dennoch ist die Arthroskopie als operativer invasiver Eingriff nicht das primäre Verfahren zur Evaluierung von Veränderungen in einem Gelenk.

Wenn sich dieses Themenheft ausführlich mit den verschiedenen nichtinvasiven Untersuchungsmethoden diverser Gelenkpathologien beschäftigt, muss darauf hingewiesen werden, dass die rein klinische Untersuchung mit ausführlicher Anamnese über den Unfallmechanismus, über Vorverletzungen und subjektive Beschwerdesymptomatik - auch im Zeitalter der technisierten Medizin - noch immer an erster Stelle stehen sollte.

\section{(7) Die rein klinische Untersuchung mit ausführlicher Anamnese sollte auch im Zeitalter der technisierten Medizin noch immer an erster Stelle stehen}

Dies wird gefolgt von Inspektion, Palpation und subtiler klinischer Funktionsdiagnostik der Gelenke im Seitenvergleich und unter Berücksichtigung der Achsenverhältnisse der betroffenen Extremität.

Die nativradiologische Abklärung eines betroffenen Gelenks schließt sich an die klinische Untersuchung an. Die Erarbeitung korrekter Bilder in primär a.-p.- und seitlicher Ebene sind essenziell und können je nach Fragestellung - Weichteil oder Knochen - in unterschiedlichen Härtegraden erstellt werden.
Die CT-Untersuchung dient im Wesentlichen zur genauen Evaluierung knöcherner Verletzungen und zur Klassifikation der Pathomorphologie der intraartikulären Frakturen sowie zur Darstellung von Osteophyten und freien Gelenkkörpern. Dadurch kann das operative Verfahren und insbesondere der Zugang genauer geplant werden und gestattet somit ein minimalinvasives Verfahren. Bei vorangegangenen operativen Eingriffen, z. B. Kreuzbandrekonstruktionen, ist die CT-Untersuchung zur Evaluierung der Tunnellage, etwaiger liegender nativradiologisch nicht sichtbarer Implantate und insbesondere Tunnelerweiterung nicht mehr wegzudenken.

Die Ultraschalluntersuchung ist - insbesondere als dynamisches Verfahren, kostengünstig und nicht strahlenbelastend für den Patienten - hat ein großes Anwendungsspektrum bei der Diagnostik der verschiedenen Sehnenpathologien ebenso wie bei Kontrolluntersuchungen nach Rekonstruktion der diversen Weichteilstrukturen. Dieses Verfahren erfordert jedoch eine ausreichende Erfahrung des Untersuchers und weist somit häufig eine große Interobservervarianz auf.

Die MRT-Untersuchung ist heute die Basis zur Indikationserstellung eines arthroskopischen Eingriffs, da nicht nur die rein intraartikulären Pathologien, sondern auch Begleitpathologien wie „bone bruise“, Knochenmarködem und extraartikuläre Weichteilstrukturen beurteilt werden können.

Im vorliegenden Themenheft stellen die einzelnen Autoren die angesprochenen diagnostischen Verfahren dar. Neben den technischen Grundlagen des 
jeweiligen Screeningverfahrens gehen die Autoren auch detailliert darauf ein, bei welcher klinischen Verdachtsdiagnose das beste nichtinvasive Verfahren zur präoperativen Diagnosesicherung angewendet werden sollte. Ableitend aus den einzelnen Beiträgen ergibt sich auch der Hinweis, wie die Fragestellung für die Radiologie $\mathrm{zu}$ formulieren ist, dass die betroffene Pathologie optimal bildgebend dargestellt werden kann.

Die postoperative radiologische Kontrolle stellt heute einen wesentlichen Dokumentationspfeiler im immer mehr juristisch werdenden medizinischen Alltag dar.

Aber auch bei postoperativ bestehenden Beschwerden und neuerlichen Traumen bei weiter zurückliegenden Eingriffen kommt der radiologischen $\mathrm{Ab}$ klärung eine immer größere Bedeutung zu. Daraus ergibt sich, dass zeitweilige gemeinsame Besprechungen mit den Radiolog(in)en eine eventuelle Rücksprache und eine detaillierte Zuweisungsanfrage wichtig sind für die Vertreter beider Fächer. Nur durch die Kommunikation können die jeweilige Befundinterpretation gebessert und das Verständnis der verschiedenen Pathologien unserer Patienten gefördert werden.

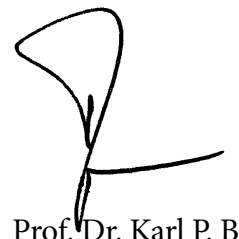

Prof.Dr. Karl P. Benedetto

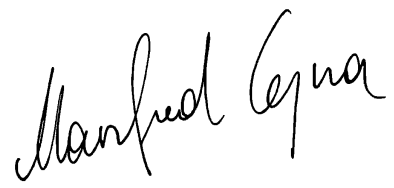

Univ.-Doz. Dr. Manfred Cejna, Msc

\section{Korrespondenzadresse Prof. Dr. K.P. Benedetto}

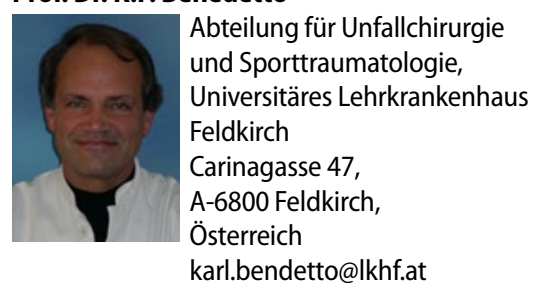

Interessenkonflikt. Der korrespondierende Autor gibt an, dass kein Interessenkonflikt besteht.

\section{Ausschreibung}

\section{Oskar und Helene-Medizin-Preis 2011}

Die Stiftung Oskar-Helene-Heim, deren Zweck die Förderung der Wissenschaft und Forschung ist, verleiht seit 2010 jährlich den mit 50.000€ dotierten Oskar und Helene-Medizin-Preis. Die Schirmherrschaft für diesen Preis hat die Bundesministerin für Bildung und Forschung, Frau Prof. Dr. Annette Schavan, übernommen. Mit diesem Medizinpreis sollen hervorragende Leistungen, insbesondere auf einem der folgenden Gebiete gewürdigt werden: Orthopädie und Orthopädie-Technik, Pneumologie sowie Gastroenterologie/Viszeralchirurgie.

\section{Der Preis wird im Jahr 2011 \\ auf dem Gebiet der}

Orthopädie und Unfallchirurgie

ausgeschrieben. Die Behandlung von nichtheilenden Frakturen stellt eine schwerwiegende Problematik dar und führt zu keinem befriedigenden Ergebnis. Im Bereich der Endoprothetik ist insbesondere nach Lockerungssituationen eine neuerliche Verankerung von Implantaten nicht möglich, da der Knochen seine Wachstumspotenz eingebüßt hat. Mit dem Preis soll daher ein habilitierter Mediziner ausgezeichnet werden, der bei der

"Stimulation der Knochenheilung bei Frakturen und / oder der Osteointegration von Endoprothesen"

besonders relevante Ergebnisse der Grundlagen- und/oder klinischen Forschung in Deutschland erzielt hat. Der prämierte Erkenntnisgewinn soll einer breiten Öffentlichkeit vermittelbar sein. Dem Antrag sollen der Lebenslauf, die fünf wichtigsten Arbeiten aus den letzten drei Jahren zum Thema „Stimulation der Knochenheilung bei Frakturen und / oder der Osteointegration von Endopro- thesen" sowie eine inhaltliche Zusammenfassung der Forschungsergebnisse beigefügt werden.

Das Preisgeld ist für Forschungszwecke nach der freien Entscheidung des Preisträgers zu verwenden. Der Preisträger hat der Stiftung diese Verwendung in geeigneter Weise zu belegen.

Der Antrag ist bis zum 30. Juni 2011 bei der Stiftung

Oskar-Helene-Heim einzureichen (www.stiftung-ohh.de).

Weitere Informationen erteilt der Geschäftsführer der Stiftung

Werner Ukas werner.ukas@stiftung-ohh.de oder info@stiftung-ohh.de

Tel. 030/8102-1100

Die Preisverleihung wird im Oktober 2011 in Berlin erfolgen.

Zweck der Stiftung Oskar-Helene-Heim ist die Förderung von Wissenschaft und Forschung in der Medizin, insbesondere der Orthopädie, der Lungenheilkunde, Gastroenterologie und Viszeralchirurgie sowie der Orthopädietechnik. Zu diesem Zweck unterstützt die Stiftung Forschungsprojekte oder sonstige gemeinnützige gesundheitsfördernde Vorhaben, verleiht Stipendien und vergibt jährlich den Oskar und Helene-Medizinpreis. Die Stiftung ist darüber hinaus Teilhaber am HELIOS Klinikum Emil von Behring im Berliner Südwesten. Seit 2004 besteht mit der HELIOS Kliniken GmbH durch die Eingliederung der damaligen Zentralklinik Emil von Behring eine strategische Partnerschaft. Gegründet wurde die Stiftung 1966. Bis zum Jahr 2000 war sie Träger des renommierten orthopädischen Krankenhauses Oskar-HeleneHeim, von 2001 bis 2004 der Zentralklinik Emil von Behring. Die Wurzeln der Stiftung reichen zurück ins Jahr 1905, als der Industrielle Oskar Pinsch gemeinsam mit seiner Ehefrau Helene einen Verein gründete, dessen Ziel die Heilung, Ausbildung und Überwachung junger Menschen mit gesundheitlichem Handicap war. 